ARTICLE

\title{
Transgenerational inheritance of impaired larval T cell development in zebrafish
}

\author{
Norimasa Iwanami13,3, Divine-Fondzenyuy Lawir ${ }^{1,4,5}$, Katarzyna Sikora', Connor O'Meara ${ }^{1}$, Kohei Takeshita², \\ Michael Schorpp ${ }^{1} \&$ Thomas Boehm (D) ${ }^{1 凶}$
}

Evidence for transgenerational inheritance of epigenetic information in vertebrates is scarce. Aberrant patterns of DNA methylation in gametes may set the stage for transmission into future generations. Here, we describe a viable hypomorphic allele of $d n m t 1$ in zebrafish that causes widespread demethylation of CpG dinucleotides in sperm and somatic tissues. We find that homozygous mutants are essentially normal, with the exception of drastically impaired lymphopoiesis, affecting both larval and adult phases of $T$ cell development. The phenotype of impaired larval (but not adult) T cell development is transmitted to subsequent generations by genotypically wildtype fish. We further find that about 200 differentially methylated regions in sperm DNA of transmitting and non-transmitting males, including hypermethylated sites associated with runx3 and rptor genes, whose reduced activities are associated with impaired larval T cell development. Our results indicate a particular sensitivity of larval $\mathrm{T}$ cell development to transgenerationally inherited epimutations.

\footnotetext{
${ }^{1}$ Department of Developmental Immunology, Max Planck Institute of Immunobiology and Epigenetics, Stuebeweg 51, 79108 Freiburg, Germany. ${ }^{2}$ RIKEN SPring-8 Center, Sayo, Hyogo 679-5148, Japan. ${ }^{3}$ Present address: Center for Bioscience Research and Education, Utsunomiya University, 350 Mine-machi, Utsunomiya, Tochigi 321-8505, Japan. ${ }^{4}$ Present address: Institute of Zoology, University of Cologne, Zülpicher Str. 47b, 50674 Köln, Germany. ${ }^{5}$ These

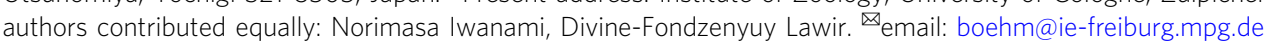


T he transgenerational inheritance of epigenetic information is an attractive mechanism by which the phenotypic consequences of exposure of parents to certain environmental conditions could be transmitted to the next generation ${ }^{1,2}$. Although this phenomenon is well described in yeast and plants, and in some invertebrates, such as D. melanogaster and C. elegans, the presence of transgenerational inheritance in vertebrates is unclear ${ }^{3}$. Among the mechanisms that are known or suspected to be involved in mediating the propagation of epigenetic information to subsequent generations, the process of enzymatic DNA methylation is of particular interest ${ }^{4-6}$. In the context of epigenetic inheritance, the induction of aberrant methylation patterns in gametes may set the stage for transmission into future generations $s^{1,2,7}$. With respect to the fate of germ cell DNA methylation patterns during early embryogenesis, important differences exist among vertebrate species. During the expansion of mouse primordial germ cells of mammals, global loss DNA methylation occurs ${ }^{8,9}$; a similar reversion to the native ground state is observed during early stages of human development ${ }^{10,11}$. Thus, the global erasure of methylation patterns in mammals constitutes a strong barrier to epigenetic memory mediated by altered patterns of DNA methylation ${ }^{12}$. By contrast, the dynamics of DNA methylation during early stages of development in zebrafish are very different and may be conducive to transgenerational inheritance. The methylome of zebrafish sperm is significantly hypermethylated when compared to that of oocytes; during the course of development, the methylome of the oocyte is gradually reprogrammed to a pattern that is similar to that of the sperm, such that by the midblastula stage, the embryo's methylation pattern is virtually identical to the sperm methylome ${ }^{13,14}$. Of note, the reprogramming process does not encompass the entire genome, but depends, at least in part, on the activity of socalled placeholder nucleosomes ${ }^{15}$. The stability of the paternal methylome throughout development ${ }^{16,17}$ raises the possibility that aberrations in the DNA methylation pattern of sperm DNA might be transmitted to the next generation.

Methylation of cytosines in DNA is established by de novo methylases Dnmt3a and Dnmt3b, whereas its propagation after DNA replication and repair depends on the maintenance methylase Dnmt1, which recognizes the hemi-methylated DNA duplex and copies the methylation pattern of the parental strand to the newly synthesized DNA strand ${ }^{18}$. Mice lacking Dnmt1 die at around day 9.5 of embryonic development ${ }^{19}$; likewise, zebrafish homozygous for a mutant $d n m t 1$ allele predicted to encode an enzyme with impaired function of the catalytic domain die at 8 days post fertilization $(\mathrm{dpf})^{20}$. These findings attest to central cellular function of Dnmt $1{ }^{18}$, but conceal a possible tissue-specific function of this protein in the adult organism. However, evidence for tissue-specific requirement of Dnmt1 has come from conditional mutants in mice (for instance, see ref. ${ }^{21}$ ), and the phenotype of zebrafish dnmt1 morphants ${ }^{22}$. However, these models fall short of providing a pan-organismic view of DNMT1 function in vertebrates.

Here, using a forward genetics approach ${ }^{23}$, we describe the identification and characterization of viable recessive allele of zebrafish dnmt1. Our results provide evidence for the transgenerational inheritance of aberrant DNA methylation patterns associated with impaired larval $\mathrm{T}$ cell development.

\section{Results}

Identification of a recessive viable allele of $\boldsymbol{d n m t 1}$. In a forward genetic screen for aberrant $\mathrm{T}$ cell development, we identified a large number of recessive mutations, all characterized by reduced numbers of rag1-expressing immature thymocytes in larvae 5 days after fertilization $(\mathrm{dpf})^{23,24}$. The mutation in the
IY071 line $\mathrm{e}^{23}$ (Fig. 1a) affected the gene encoding the maintenance DNA methyltransferase DNMT1. The recessive viable dnmt1 allele $\left(d n m t 1^{\mathrm{t} 25501}\right)$ exhibits a missense mutation $(\mathrm{N} 1391 \mathrm{~K})$ in the target recognition domain (TRD) of the catalytic domain of the enzyme (Fig. 1b) ${ }^{23}$; since a previously identified dnmt1 mutant allele is embryonic lethal ${ }^{20}$, we consider the $d n m t 1^{125501}$ allele to be a hypomorph. The mutation in dnmt $1^{\text {t25501 }}$ occurs in an evolutionarily conserved region of the protein (equivalent to N1510K in the mouse protein) (Fig. 1c), and affects an amino acid residue whose side chain in the mouse protein projects towards the major groove of the substrate DNA ${ }^{25,26}$ (Fig. 1d).

Hypomethylation of DNA in dnmt1 mutants. Fish homozygous for the N1391K mutation exhibit drastically reduced levels of cytosine methylation in $5^{\prime}-\mathrm{CpG}$ dinucleotides of genomic DNA. In DNA extracted from wild-type whole fish at 18 days after fertilization (dpf), 89.08\% (median; median absolute deviation [mad] 8.79\%) of CpG dinucleotides are methylated whereas the methylation levels in mutants are much lower reaching a mere $56.72 \%$ (median; mad 23.04\%) (Fig. 1e). The methylation levels in sperm DNAs are generally higher than in somatic tissues, reaching $97.62 \%$ (median; mad 3.09\%) in the wild-type situation; in $d n m t 1^{\mathrm{m}} / \mathrm{m}$ males, in which germ cells develop in the absence of zygotically provided dnmt1 protein, methylation levels fall to 71.81\% (median; mad 21.52\%) (Fig. 1f). The extent of hypomethylation is more pronounced in DNA extracted from whole body as compared to mutant sperm, falling to $63.6 \%$ and $73.6 \%$ of wild-type values, respectively (Fig. 1g). Remarkably, despite the dramatic hypomethylation of somatic DNA, dnmt 1 hypomorphs are viable, making this zebrafish strain an unprecedented model with which to explore the physiological consequences of low DNA methylation levels in an organismic context.

Hematopoietic abnormalities in dnmt1 mutants. Next, we examined the hematopoietic system in $d n m t 1^{\mathrm{m} / \mathrm{m}}$ animals during larval and adult stages (Fig. 2a). At $5 \mathrm{dpf}$, no changes were recorded for the expression levels of markers for hemogenic endothelium (gata2b) and haematopoietic stem cells $(c-m y b)$. Slightly reduced levels were found for genes indicative of erythroid (gata1a) and myeloid (cebpa) lineages, although expression of $m p x$ as a marker of mature myeloid cells was more substantially reduced (Fig. 2b). At this stage of development, the most drastic changes in expression were seen for genes associated with $\mathrm{T}$ cell development $(l c k ; z a p 70)$ and for genes whose expression is associated with, but not restricted to, developing T cells (rag1; ikzf1; gata3) (Fig. 1a; Fig. 2b). Next we examined the status of adult hematopoiesis in whole kidney marrow. Among the cell populations discernable by their light-scatter characteristics, the most consistent reduction was seen for lymphocytes, whereas erythroid and myeloid cell populations were not affected (Fig. 2c). In order to substantiate this conclusion, we introduced an ikzf1:egfp transgene ${ }^{27}$ into the IY071 line; in adult fish, the reporter marks $\mathrm{T}$ and $\mathrm{B}$ cells. As suggested by the aberrant flow cytometric profile, the lymphocyte population is greatly diminished in $d n m t 1^{\mathrm{m} / \mathrm{m}}$ fish (Fig. 2c). Collectively, our results suggest the lymphopoietic capacity of adult $d n m t 1^{\mathrm{m} / \mathrm{m}}$ animals is drastically impaired, indicating that the genetically separable waves of larval and adult $\mathrm{T}$ cell development in zebrafish ${ }^{28,29}$ (Fig. 2a) are equally affected.

Hematopoietic abnormalities in $\boldsymbol{d n m t 1}$ mutant offspring. We found that the offspring (designated as generation 3 [G3] in Fig. 3a) of male mutant fish crossed with wild-type females are viable, as are those of male mutant fish crossed with heterozygous females. Therefore, it was possible to examine the hematopoietic 
a

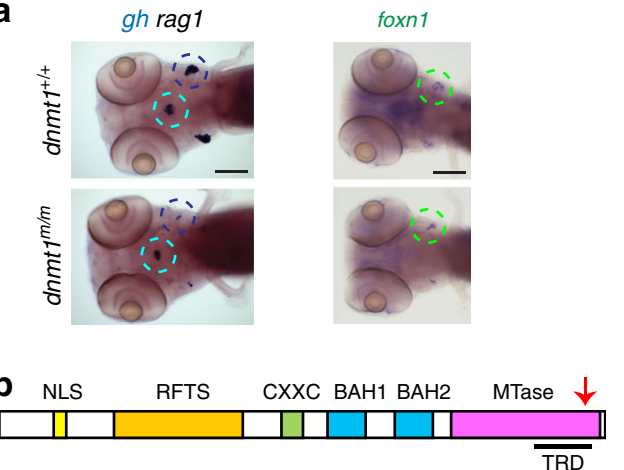

C $\begin{array}{ll}\text { D.r. } & <\text { dRQFnTLIPWCLPHTGNRHNHWAGLYGRLEWDGFFSTTVT> } \\ \text { M.m. } & <\text { SRQFSTLIPWCLPHTGNRHNHWGLYGRLEWDGFFSTTVT> }\end{array}$ N1510

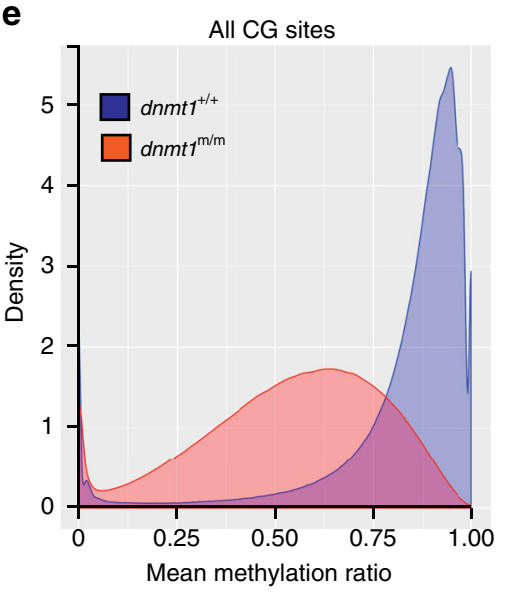

$\mathbf{f}$

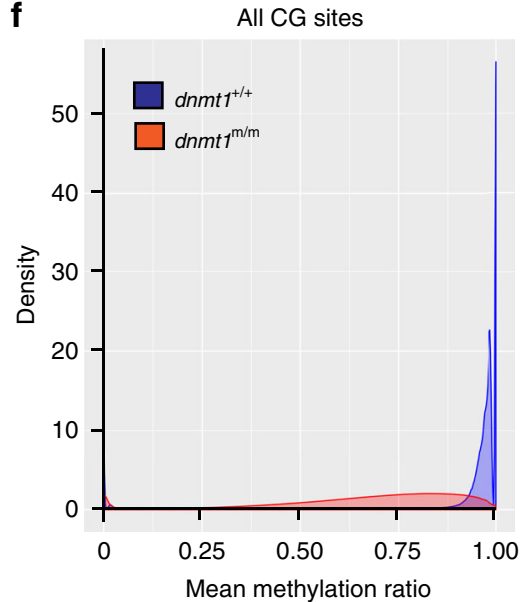

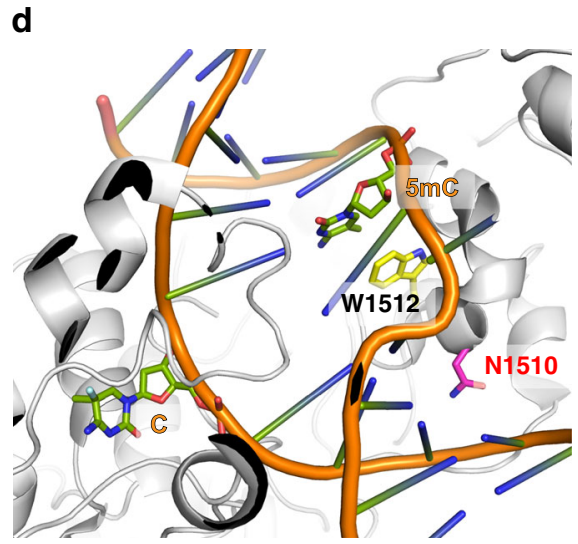

g

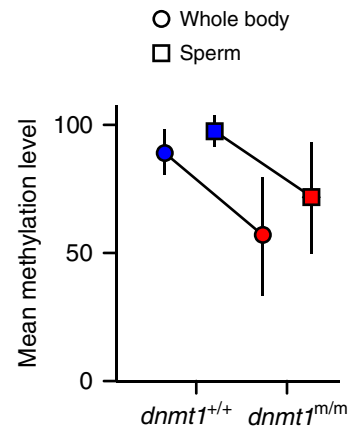

Fig. 1 Characterization of a hypomorphic allele of dnmt1. a Impaired larval T cell development in mutant fish. Diagnostic whole-mount RNA in situ hybridization pattern ${ }^{23}$ in wild-type and dnmt 1 mutant fish at $5 \mathrm{dpf}$ using rag1 (thymus encircled in black), and gh (hypophysis encircled in blue) specific probes (left panel); hybridization pattern for foxn1, a marker of thymic epithelium 59,60 (right panel). Scale bar, $100 \mu \mathrm{m}$. Panels are representative of 25 animals each. b Schematic of functional domains in the dnmt1 protein (not to scale); NLS, nuclear localization signal; RFTS, replication foci targeting site; CXXC, cysteine-rich domain; BAH, bromo-adjacent homology domains 1 and 2; MTase, catalytic domain. Arrow, approximate position of the amino acid replacement in the target recognition domain, TRD. c The asparagine residue mutated in the dnmt $1^{25501}$ allele occurs in an evolutionarily conserved region of the enzyme. $\mathbf{d}$ In the structure of the mouse Dnmt1 protein in complex with a hemi-methylated substrate, close apposition of the mutated asparagine residue to the substrate DNA in the catalytic site is observed ${ }^{26}$ (PDB ID: 4DA4). e, $\mathbf{f}$ Hypomethylation of cytosine residues in CpG dinucleotides of DNA extracted from whole body of $18 \mathrm{dpf}$ larvae $(\mathbf{e})(n=3)$ and sperm of adult homozygous mutants $(\mathbf{f})(n=3)$. In $(\mathbf{e})$ and $(\mathbf{f})$, density refers to the fraction of CG sites with a particular methylation ratio. $\mathbf{g}$ Mean methylation levels of CG dinucleotides for DNAs shown in (e) and (f). Values shown represent mean $\pm \operatorname{mad} ; n=3$. Source data are provided as Source Data file.

compartments of larval and adult stages of G3 fish. In contrast to heterozygous G2 animals arising from a cross of heterozygous males and females, we observed that many of the heterozygous G3 animals resulting from a cross of homozygous mutant males with wild-type females (Fig. 3a) exhibited impaired larval T cell development (Fig. 3b, c). By contrast, the adult $\mathrm{T}$ cell compartment of these heterozygous G2 and G3 fish develops normally (Fig. 3d). The striking discrepancy between the hematopoietic phenotypes of G2 and G3 fish suggests that the functionally distinct phases of $\mathrm{T}$ cell development in zebrafish are not only genetically ${ }^{28,29}$, but also epigenetically separable. Moreover, the present results suggest a particular sensitivity of larval $\mathrm{T}$ cell development to aberrations of DNA methylation.

The unexpected finding of impaired larval $\mathrm{T}$ cell development in heterozygous fish arising from crosses of mutant males and wild-type females prompted us to extend the analysis to include further generations (Fig. 4a). To this end, the G1 mutant carrier was out-crossed to wild-type fish twice, to establish two separate pedigrees. The extent of larval and adult $\mathrm{T}$ cell development was examined at each generation, but no differences were observed between these two separate populations. We found that in contrast to the situation of G2 mutant males, the progeny of a cross of G2 mutant females with wild-type males succumbed to an early developmental arrest before body axis formation. During early development, the maternal methylome is gradually reprogrammed to the paternal methylation pattern ${ }^{13-15,30}$, indicating that the enzymatic activity of the mutant Dnmt1 protein deposited in the eggs is not sufficient to support this remodeling. Because full Dnmt1 activity appears to be required before the onset of zygotic gene activation, only mutant males could be used to establish heterozygous G3 progeny; these fish were crossed to wild-type fish to generate $d n m t 1^{+/+}$and $d n m t 1^{+/ m}$ animals of the G4 generation (Fig. 4a). When we crossed male or female fish of the G4 generation that are genotypically wildtype for $d n m t 1$ with wild-type females or males (Fig. 4a), G5 fish with strikingly reduced larval $\mathrm{T}$ cell development were observed in $\sim 50 \%$ of the 
a
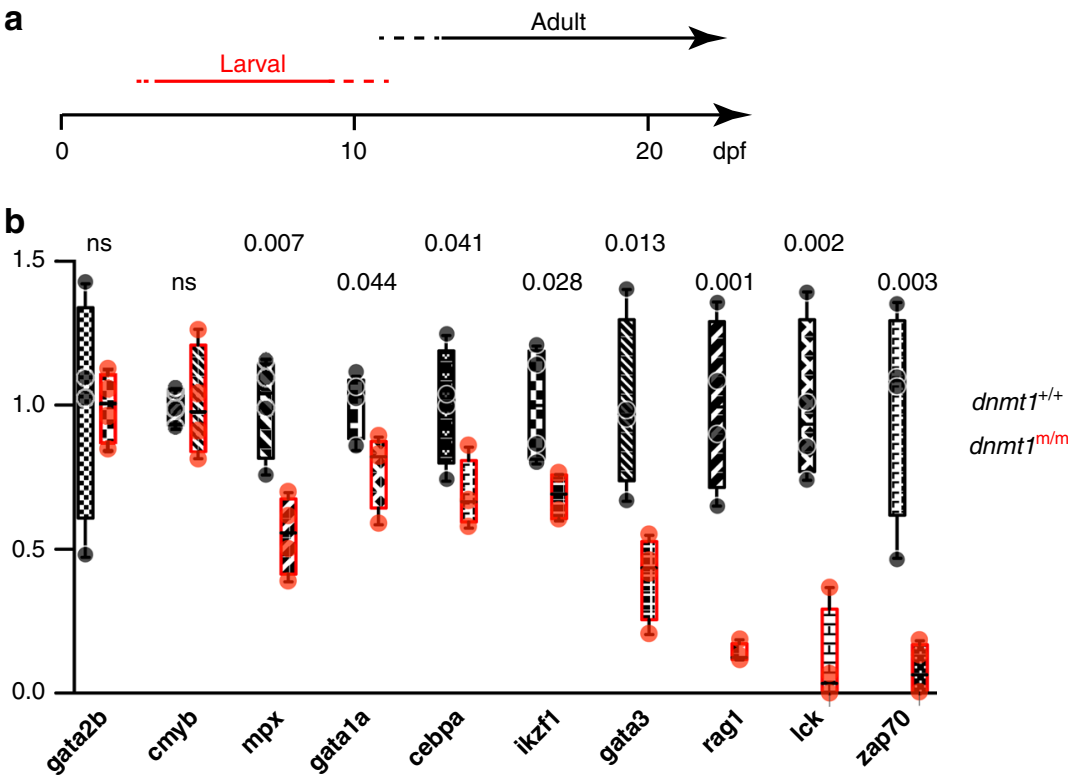

C
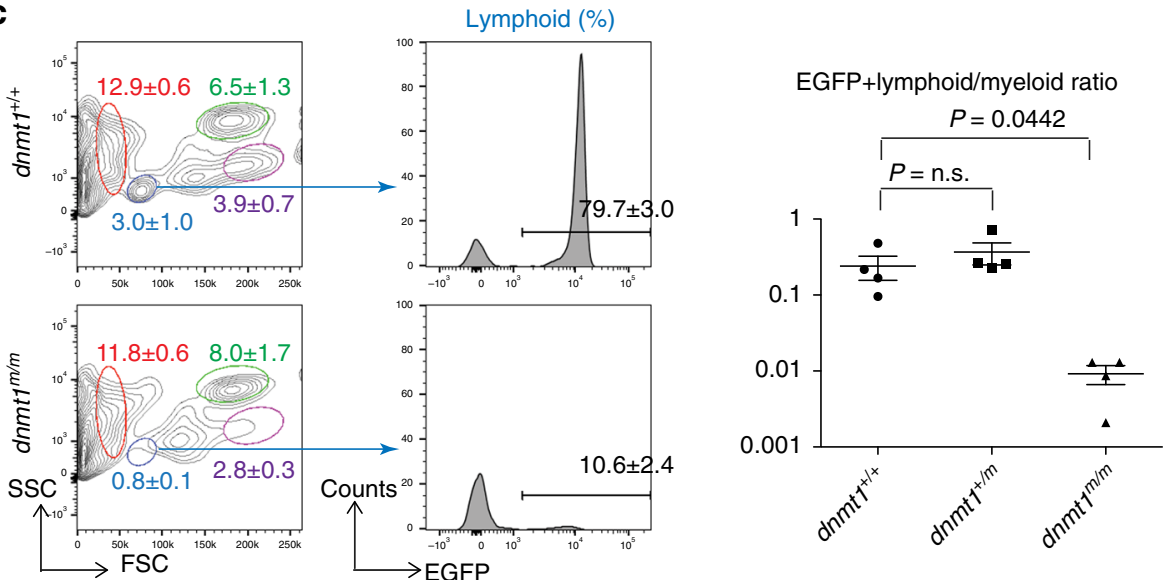

Fig. 2 Hematopoietic abnormalities in dnmt1 mutant fish. a Schematic indicating the two waves of T cell development in zebrafish, operationally referred to as larval and adult phases; the larval phase depends on the activity of the ikzf1 transcripton factor 28,46 . In the forward genetic screen, fish were analyzed at $5 \mathrm{dpf}$. b Expression patterns of selected genes associated with hematopoietic development in $5 \mathrm{dpf}$ G2 embryos. Levels were determined by qPCR and normalized to the levels of $a c t b 1$ ( $n=4$; mean; whiskers represent maximum and minimum values; individual data points are indicated). c Adult lymphopoiesis fails in mutant fish. Flow cytometric analyses of whole kidney marrow (WKM) cells ${ }^{47}$ of wild-type and dnmt1 mutants, both transgenic for an ikzf1-EGFP reporter 27,28 (left panels). Circles denote different blood cell populations in adult wild-type fish: red, erythrocytes; blue, lymphocytes; magenta, precursors; green, myelomonocytes. Percentages (mean \pm SD) of each population in WKM preparations are indicated $(n=4$, for both genotypes; left panels). Analysis of EGFP-positive cells in the lymphocyte gates; percentages of positive cells are indicated in the histograms ( $n=4$, for both genotypes; middle panel), and enumerated (right panels). FSC, forward light scatter; SSC, side light scatter. Source data are provided as Source Data file. For b, c, unpaired two-tailed $t$ test with Welch's for unequal variance and Bonferroni's correction for multiple tests.

crosses (Fig. 4b); this phenotype was observed in both pedigrees, arguing against the fortuitous co-segregation of an unknown mutation. Henceforth, G4 fish that yield normal offspring are designated as $\mathrm{G}^{+}$, whereas those that sire offspring with impaired larval $\mathrm{T}$ cell development are designated as $\mathrm{G} 4 *$. As expected, the cellular composition of the hematopoietic cell populations in the kidney marrow was normal for genotypically wild-type adult males and females that gave rise to offspring with impaired larval T cell development (Fig. 4c).

Transgenerational inheritance of larval $T$ cell impairment. In G4* $\mathrm{x}$ wild-type crosses, $\sim 1 / 3$ of fish of the clutch presented with $\mathrm{T}$ cell deficiency; this phenomenon was observed in both male (Fig. 5a) and female (Fig. 5b) G4 animals. Indeed, when the range of rag1/gh values observed in the resulting G5 off-spring is determined and expressed as "noise" (variance normalized to the mean), $4^{+}$and $\mathrm{G} 4 *$ animals can be clearly distinguished; G4* animals exhibit a 7 -fold higher noise in rag1/gh values (Fig. $5 \mathrm{c}$ ). The phenomenon of reduced larval $\mathrm{T}$ cell development in genotypically wild-type G5 fish was also seen in crosses of $d n m t 1^{+/ \mathrm{m}}$ G4 males with wild-type females, again accompanied by increased noise in the ragl/gh ratios in the two resulting genotypes (Fig. 5d). Collectively, these data suggest that the transgenerational inheritance of an aberrant DNA methylation mark(s) may underlie the aberrant larval $\mathrm{T}$ cell development.

In order to exclude the possibility that this phenomenon is the result of non-specific demethylation of the parental genomes, we repeated the crosses using wild-type parents treated with 5-aza$2^{\prime}$-deoxcytidine (aza-dC), an irreversible inhibitor of DNA 


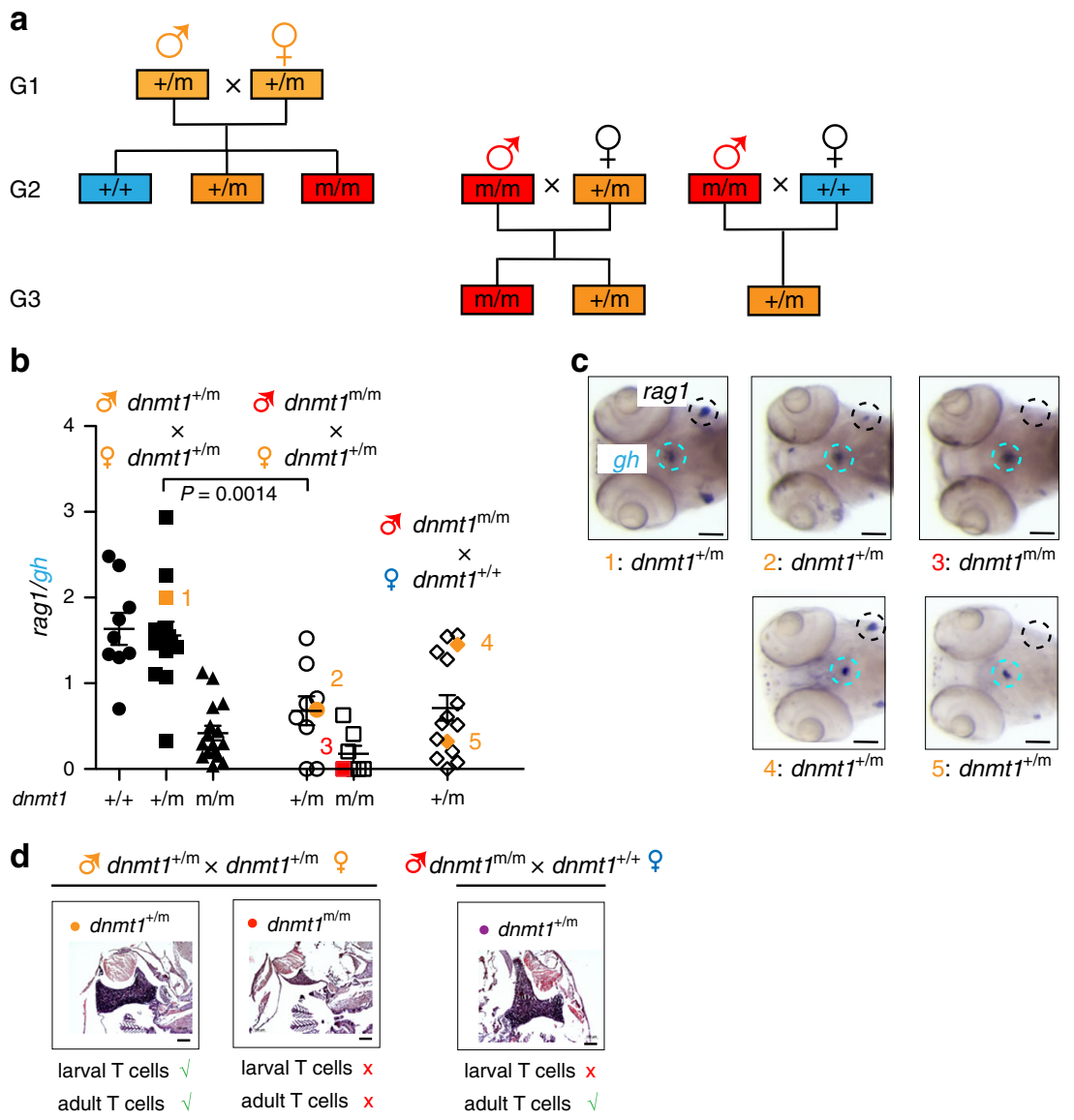

Fig. 3 Impaired T cell development in $\mathbf{5}$ dpf larvae. a Structure of pedigrees. A cross of male and female carriers gives rise to the three genotypes of the G2 generation that are used to establish the G3 generation. b The rag1/gh ratio as determined by RNA in situ hybridization is shown for 5 dpf fish of the indicated genotypes (bottom) arising from the indicated parental genotypes (top); male genotypes are shown in upper row. Each data point represents one animal; $t$ test, two-tailed; mean \pm s.e.m. c Representative RNA in situ patterns on which the calculation of rag $1 / g h$ ratios was done are shown on the right for the animals identified by numbers in panel (b). The thymus region (identified by ragl signal) and the hypohysis (identified by the $g h$ signal) are encircled; because of the cell-type specificity of the mutant phenotype, the rag1/gh ratio serves as a convenient normalization measure for the RNA in situ hybridization protocol. d Thymopoiesis in adult animals of the indicated generations and genotypes. Histological sections of thymi were stained with hematoxylin/eosin; note the alymphoid thymus in $d n m t 7^{\mathrm{m}} / \mathrm{m}$ fish. $\ln (\mathbf{c}, \mathbf{d})$, scale bars: $0.1 \mathrm{~mm}$. Source data are provided as Source Data file.

methylases $^{31}$. However, while aza-dC treatment reduced the extent of larval $\mathrm{T}$ cell development, no abnormalities were observed at later stages (Supplementary Fig. 1); most importantly, the adults exposed to the demethylating agent during the embryonic period did not produce offspring with aberrant $\mathrm{T}$ cell development, suggesting that the particular nature of changes in methylation patterns associated with the N1391K mutation underlies the transgenerational phenotype.

Characterization of DNA methylation patterns in G4 sperm. Next, we analyzed the methylation patterns of G2 wild-type and mutant sperm. In the DNA of G2 $d n m t 1^{\mathrm{m} / \mathrm{m}}$ sperm, we detected 223,956 hypomethylated and 175 hypermethylated differentially methylated regions (DMRs); for simplicity, we refer to the methylation pattern in G2 mutant sperm as pattern A. Interestingly, G2 fish homozygous for the dnmt1 mutation exhibit increased expression levels of the de novo DNA methyltransferase $d n m t 3 b b .2$ at $5 \mathrm{dpf}$, suggesting the activation of compensatory mechanism(s) partially counteracting impaired maintenance activity of dnmt1; only minor differences were noted for other genes encoding modulators of DNA methylation (Supplementary Fig. 2a).

We then analyzed the DNA methylation patterns of sperm of the two groups of genotypically wild-type G4 males, $4^{+}$and
G4*, respectively, and found that they differed by a mere 243 DMRs; 164 DMRs are hypomethylated in G4* (Supplementary Data 1), and 79 DMRs are hypermethylated in G4* sperm (Supplementary Data 2); we refer to the methylation patterns in G4 mutant sperm as patterns $\mathrm{B}^{+}$and $\mathrm{B}^{*}$, respectively. For for $\mathrm{G}^{+}{ }^{+}$sperm, the degree of methylation of $\mathrm{CpG}$ dinucleotides across the genomes is $97.57 \%$ (median; mad 2.99\%); for G4*, this value is $97.53 \%$ (median; mad $3.02 \%$ ) (Fig. 6a); these methylation levels are very close to those of wild-type sperm (Fig. 1f). The vast majority of DMRs identified in the comparison of $\mathrm{G}^{+/+}$and $\mathrm{G} 2 \mathrm{~m} / \mathrm{m}$ sperm could also be evaluated in G4 sperm. Of the 222,449 hypomethylated G2 DMRs, the methylation levels of 222,390 DMRs (99.3\%) were identical between $\mathrm{G}^{+}{ }^{+}$and G4* sperm DNAs; of the 171 hypermethylated G2 DMRs, 170 DMRs (99.4\%) were no longer distinguishable in G4 sperm. Collectively, our observations suggest that during the transition from G2 to G4, the germ cells developing in the presence of zygotically provided dnmt1 protein undergo global restoration of methylation patterns.

Next, we examined the history of the DMRs distinguishing $\mathrm{G}^{+}{ }^{+}$and $\mathrm{G} 4^{*}$ sperm DNAs (Fig. 6b). Of the 164 hypomethylated DMRs, 162 DMRs were also observed in the G2 comparison and could thus be included in our analysis; 101 DMRs were still hypomethylated in $\mathrm{G}^{*}$ sperm, whereas a further 61 DMRs that 
a

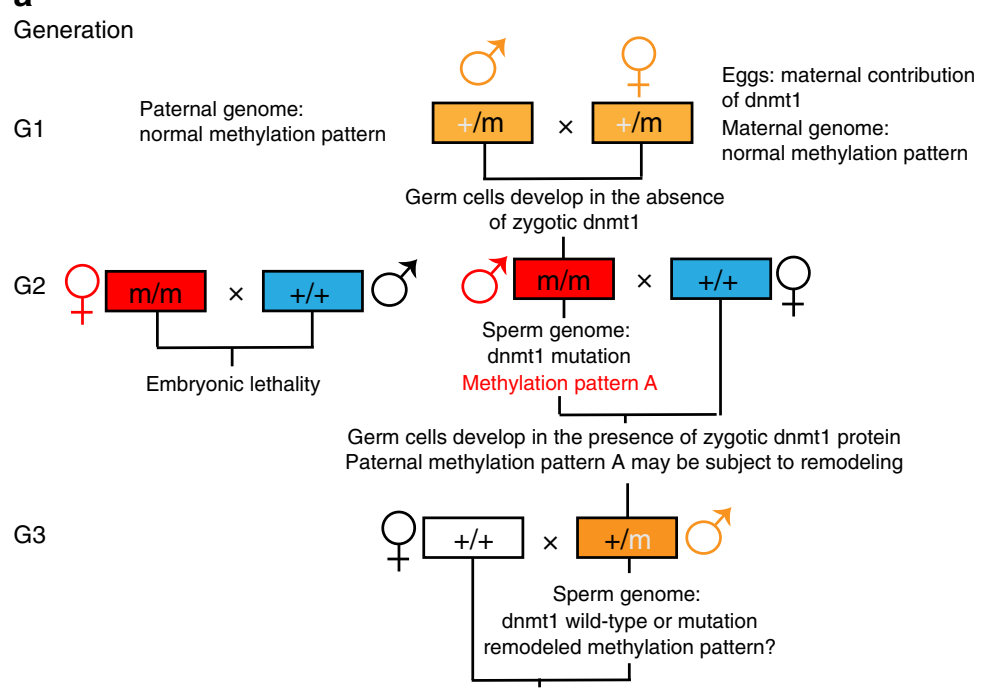

Germ cells develop in the presence of zygotic dnmt1 protein remodeled paternal methylation pattern?

G4

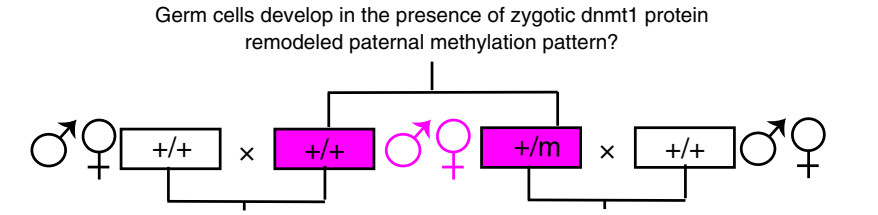

G5

Germ cells develop in the presence of zygotic dnmt1

Parental methylation pattern $B$ is established

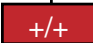

Analysis in Fig. 5a-c

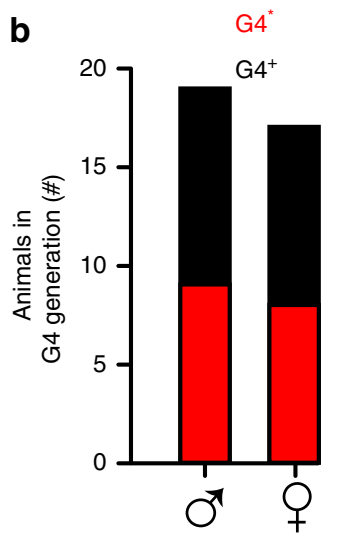

Analysis in Fig. 5d
T cell development

Larval Adult

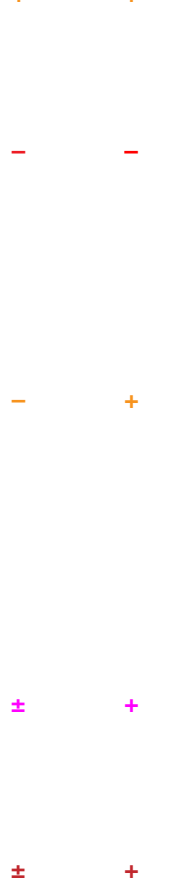

C

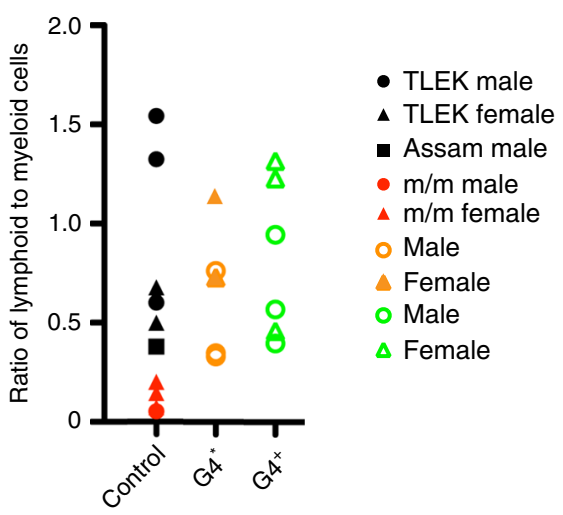

Fig. 4 Transgenerational inheritance of failing larval T cell development. a Structure of pedigree; the genotypes and sex of animals is indicated. White boxes, wild-type animals unrelated to the dnmt7-mutant lineage; blue boxes, genotypically wild-type animals arising from various crosses in the dnmt1mutant lineage. The presence $(+)$ or absence $(-)$ of larval and adult phases of T cell development in different generations of the pedigree are indicated on the right, with colors matching the relevant genotypes. The \pm sign indicates that larval T cell development is impaired in some but not all G4 and G5 animals. b Genotypically wild-type $\left(d n m t 7^{+/+}\right)$males and females of the $\mathrm{G} 4$ generation give rise to off-spring with $\left(\mathrm{G} 4^{\star}\right)$ or without $\left(\mathrm{G} 4^{+}\right)$impaired larval T cell development when crossed to wild-type animals. c Normal adult hematopoiesis in fish of different genetic backgrounds. Whole kidney marrow cells were analyzed by flow cytometry (see Fig. 2c, left panel) and cell numbers in the lymphoid and myeloid gates were counted ${ }^{47}$. The genotype of animals is indicated to the right; TLEK and Assam represent wild-type strains. Note the normal lymphoid/myeloid ratios in adult G4^ animals. Each data point represents one animal. Source data are provided as Source Data file.

were originally indistinguishable in G2 exhibit lower methylation levels in G4* (Fig. 6b; Supplementary Data 3). Of the 79 hypermethylated DMRs distinguishing $\mathrm{G}^{+}$and $\mathrm{G} 4^{*}$ sperm DNAs, the status of 75 DMRs could be assessed in G2; 58 of these DMRs exhibited similar methylation levels in wild-type and mutant G2 sperm, whereas 17 DMRs were found to have lower methylation levels in $\mathrm{G} 2 \mathrm{~m} / \mathrm{m}$ sperm as compared to wildtype (Fig. 6b; Supplementary Data 3). The DMRs distinguishing G4+ and G4* sperm DNAs are non-randomly distributed across the genome (Fig. 6c), with chromosomes 6, 9, 10, and 16 being particularly affected (Fig. 6d).

Gene regulatory context of DMRs. Next, we wished to gain sight into the distribution of DMRs in G2 and G4 with respect to known gene regulatory elements. We found that $\mathrm{CpG}$ islands, 
a
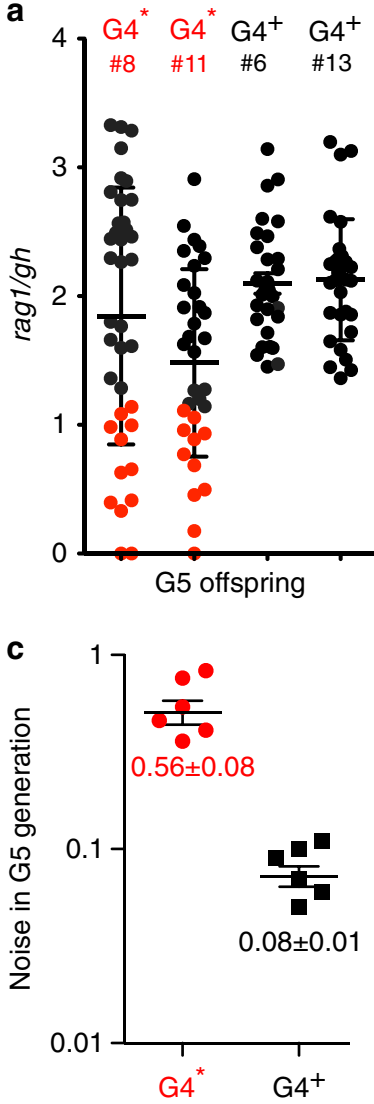

b

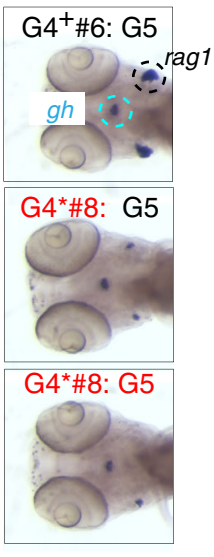

d

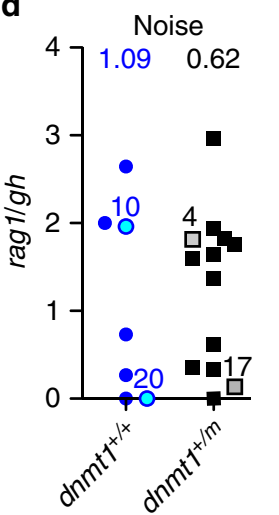

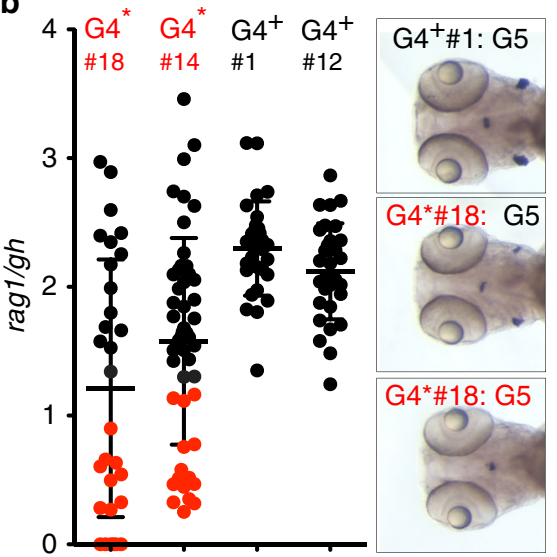

G5 offspring

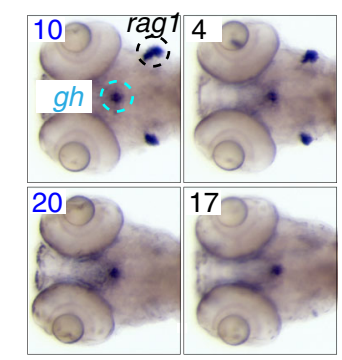

Fig. 5 Variable levels of larval T cell development in the G5 generation. a Extent of T cell development (expressed as ragl/gh ratio) in the G5 generation arising from dnmt1+/+ male G4 fish (c.f., a). Red dots represent values of thymopoietic index of animals in G5 clutches arising from a G4* parent whose $\mathrm{rag} / \mathrm{gh}$ ratios lie outside 2 standard deviations of values observed in G5 clutches arising from G4+ parents. Representative RNA in situ results are shown for $\mathrm{G} 5$ fish. $\mathbf{b}$ Extent of $\mathrm{T}$ cell development in the $\mathrm{G} 5$ generation arising from $d n m t \mathrm{~T}^{+/+}$female $\mathrm{G} 4$ fish. c Noise (calculated as variance/mean) values of rag1/gh data in $\mathrm{G} 5$ clutches arising from $4^{\star}$ and $4^{+}$parents (mean \pm s.e.m.).d Extent of T cell development (expressed as rag1/gh ratio) in the G5 generation arising from $d n m t 1^{+/ m}$ male G4* fish; the mean noise values are indicated at the top (left panel). Representative RNA in situ patterns on which the calculation of rag1/gh ratios was performed are shown on the right for the animals identified by numbers in the left panel. In (a-d), each data point represents one animal. Source data are provided as Source Data file.

promoters, exons, introns and intergenic regions were similarly affected by the extensive changes in methylation of G2 mutant sperm DNA. Moreover, none of these features was associated with the methylation differences observed between $\mathrm{G}^{+}$and $\mathrm{G} 4^{*}$ sperm DNAs (Supplementary Fig. 2b). As expected, genomic regions marked by placeholder nucleosomes ${ }^{15}$ were found to be mostly unmethylated, and no changes in methylation patterns at these sites were observed in any of the experimental groups. Moreover, DMRs exhibiting dynamic changes during early zebrafish development, such as those changing between the epiboly stage and the $24 \mathrm{hpf}$ time point, and those changing between the $24 \mathrm{hpf}$ time point and the $48 \mathrm{hpf}$ time point ${ }^{32}$ also could not distinguish $\mathrm{G}^{+}$and G4* sperm DNAs (Supplementary Fig. 2c). The analysis of repetitive sequences indicated that all classes of repeats were uniformly affected by hypomethylation in G2 mutant sperm DNA; in G4 sperm, all repeats participated in the restoration of methylation levels equally well (Supplementary Fig. 2d).

We then asked whether any particular chromatin signature would explain the differences between the methylation patterns of $\mathrm{G}^{+}$and $\mathrm{G} 4^{*}$ sperm DNAs and examined the enrichment of specific chromatin marks at the hypo- and hypermethylated G4 DMRs. None of the distinct DNA methylation patterns could be explained by histone modification combinations (Supplementary
Fig. 3). Since we observed a disproportionately high number of G4 DMRs on chromosome 6 (Fig. 6c, d), we investigated DMR distribution in context of chromosome-scale features. To this end, we compared chromosome 6 (large number of DMRs discovered) with chromosome 7 (no DMRs discovered) (Supplementary Fig. 4). We found that neither the positions of topologically associated domains (TADs), nor the presence of specific chromatin marks (H3K4me3; H3K27me3; H3K9me3), CpG islands, or repetitive sequences were associated with clusters of hypo- and hypermethylated DMRs (Supplementary Fig. 4). Further studies are required to determine the mechanistic basis of the uneven distribution of DMRs distinguishing the methylation patterns of $\mathrm{G}^{+}$and $\mathrm{G} 4^{*}$ sperm DNAs.

Characterization of hypermethylated DMRs in G4* sperm. Our results suggest that the hematopoietic abnormalities of G2 mutants have their origin in a progenitor common to both $\mathrm{T}$ and B lymphoid lineages in larvae and adult fish. By contrast, G4* animals give rise to a more circumscribed aberration in their offspring, originating in the incapacitation or lack of a larval $\mathrm{T}$ cell progenitor only. This in turn suggests that the failure of $\mathrm{T}$ cell development observed in G4* progeny may have a different or only partially overlapping epigenetic basis than that in earlier 
a

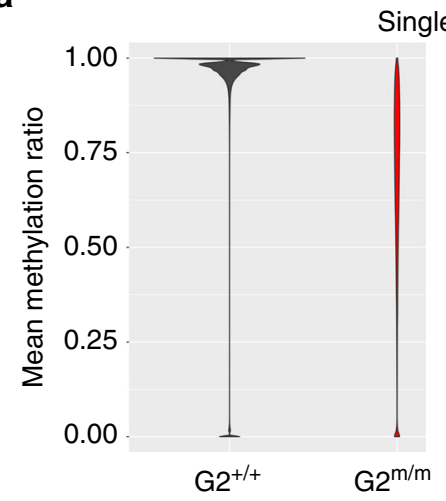

Single CpG sites

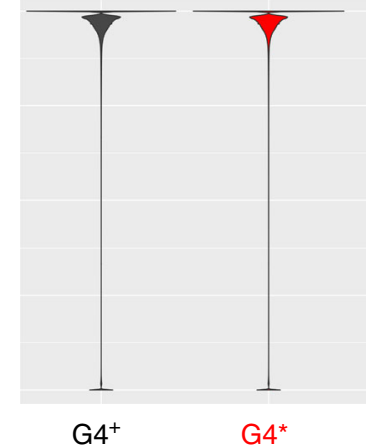

$\mathrm{G}^{+}$ $\mathbf{b}_{250}$

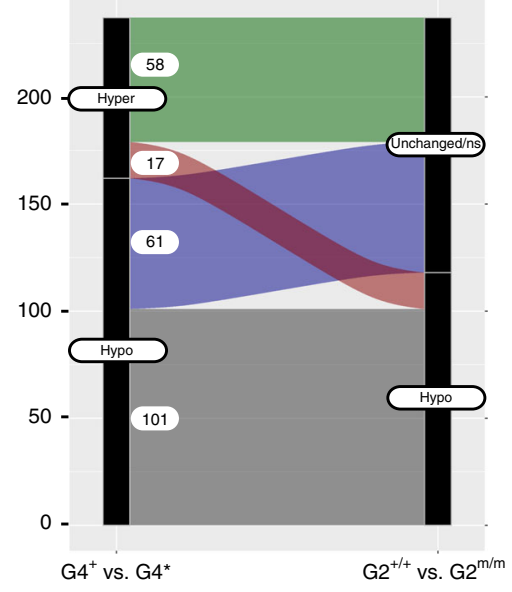

C

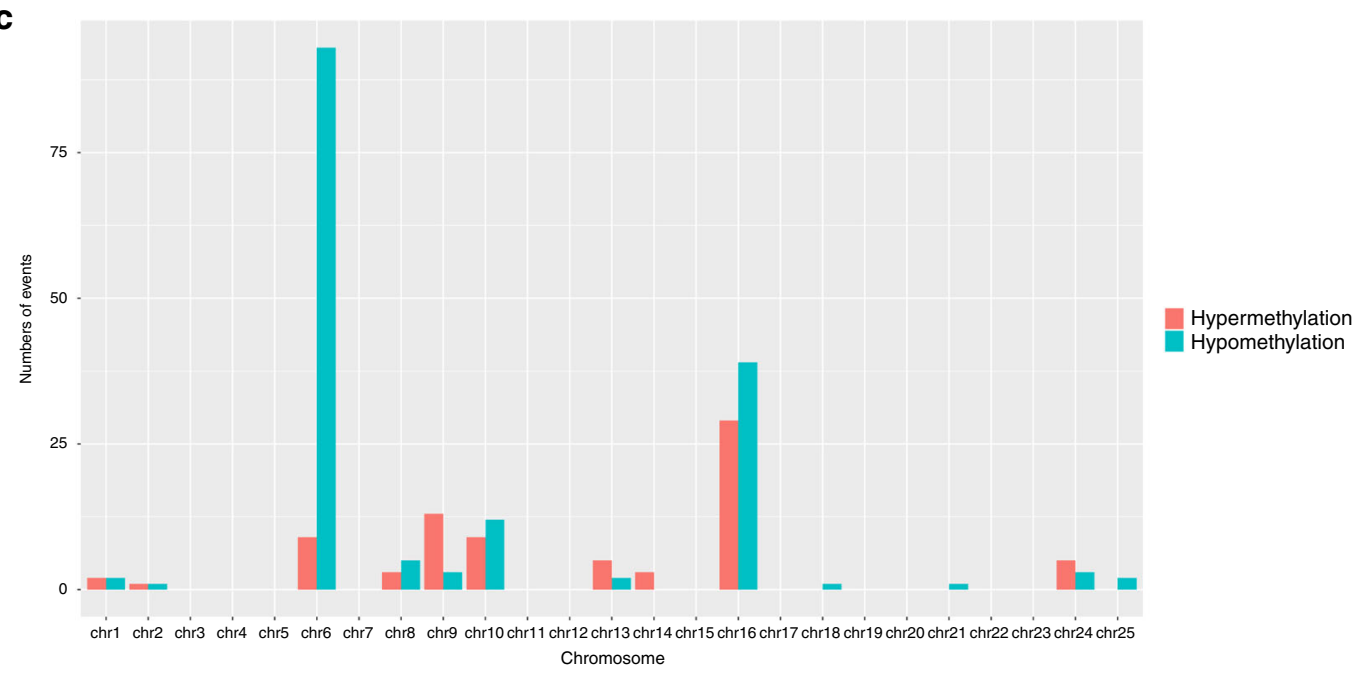

d
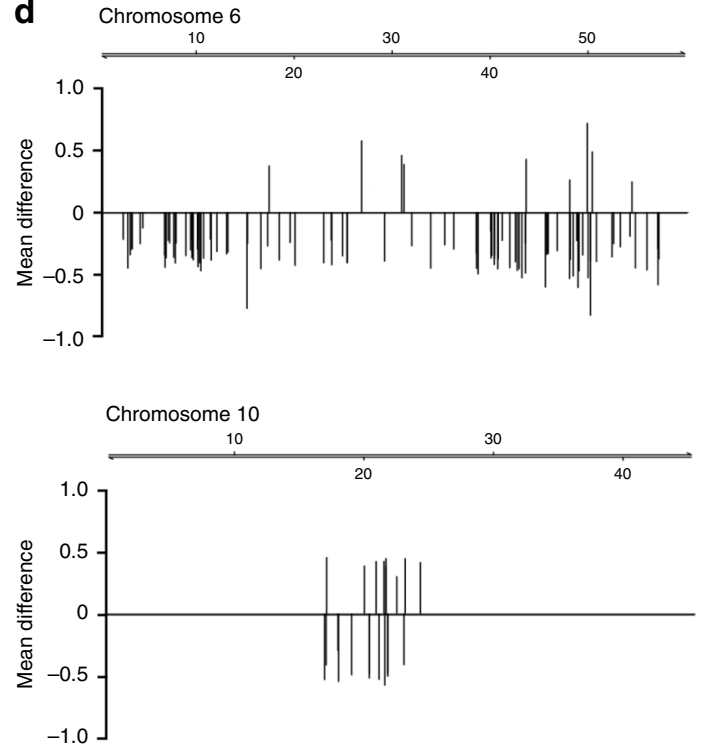
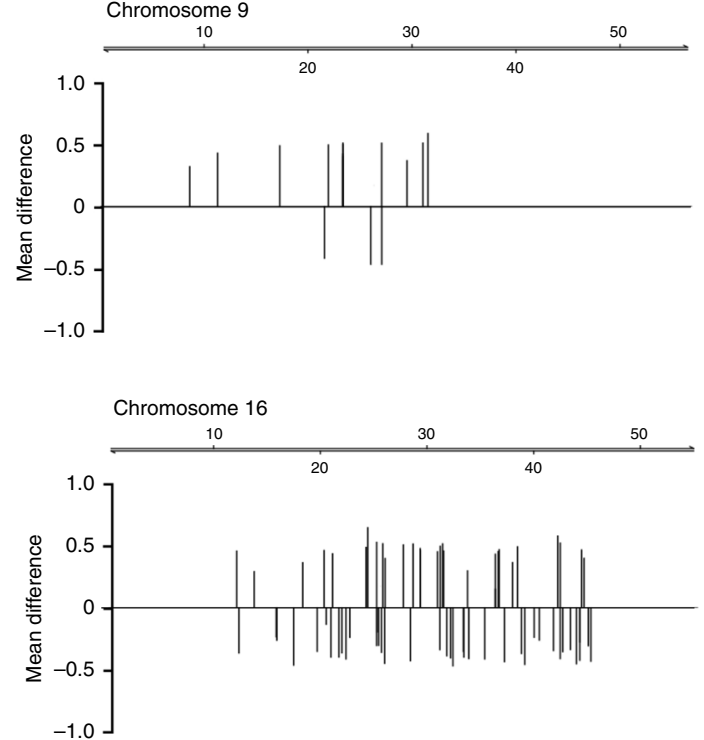

genes, respectively (Fig. 7a, b; Supplementary Fig. 5a, b); previous studies in mice have shown that these genes are both required for intrathymic $\mathrm{T}$ cell development ${ }^{33,34}$. In both cases, the hypermethylated DMR overlaps with a region distinguished by chromatin marks, such as $\mathrm{H} 3 \mathrm{~K} 4 \mathrm{me} 1, \mathrm{H} 3 \mathrm{~K} 4 \mathrm{me} 3$, and $\mathrm{H} 2 \mathrm{AFV}$, indicative of regulatory function $(s)^{15}$. Although we consider it generations. Hence, we focused our attention on those DMRs in G4 sperm whose methylation levels are discordant to G2 mutant sperm (Fig. 6b; Supplementary Data 3). To this end, we searched for DMRs associated with genes known to be important for T cell development. Notably, in $\mathrm{G} 4 *$ sperm, we identified one hypermethylated DMR each that is associated with runx3 and rptor 
Fig. 6 Dynamic changes of DNA methylation patterns in the $\mathbf{G 4}$ generation. a Mean methylation ratios of CpG dinucleotides in sperm DNAs of G2 $d n m t 7^{+/+}$and $d n m t 7^{m} / m$ animals (left panel), and genotypically wild-type $\left(d n m t 1^{+/+}\right.$) males of the G4 generation giving rise to off-spring with (G4*) or without $\left(\mathrm{G}^{+}\right)$impaired larval T cell development when crossed to wild-type females (right panel). b History of methylation of DMRs distinguishing G4+ and $\mathrm{G} 4^{\star}$ animals. The $y$-axis gives the number of DMRs that are either hypermethylated or hypomethylated in sperm DNA of in G4* versus G4+ animals. These DMRs were either indistinguishable in sperm of the $\mathrm{G} 2$ generation or were hypomethylated in $\mathrm{G} 2^{\mathrm{m} / \mathrm{m}}$ versus $\mathrm{G} 2^{+/+}$animals. c, d Non-random distribution of DMRs in the genome of sperm of $\mathrm{G} 4^{\star}$ relative to $\mathrm{G}^{+}{ }^{+}$animals. Most DMRs represent hypomethylated sites $\mathbf{c}$. Distribution of DMRs across the 4 most affected chromosomes (scale in $\mathrm{Mb}$ ); the mean methylation differences are indicated; positive values indicate hypermethylation of DMRs in G4* sperm, negative values indicate hypomethylation of DMRs in $4^{\star}$ sperm (d). Source data are provided as Source Data file.

likely that genes other than runx3 and rptor and/or their concerted activities contribute to the observed T cell phenotype in the offspring of G4* animals, we found that simultaneous knockdown of these two genes led to impaired larval $\mathrm{T}$ cell development (Fig. 7c). Moreover, despite the fact that the expression of runx 3 and rptor are not exclusive to the $\mathrm{T}$ cell lineage, a trend towards lower expression levels of both genes was observed in $5 \mathrm{dpf}$ mutant larvae (Supplementary Fig. 5c). Collectively, our findings suggest that runx3 and rptor are functionally relevant for zebrafish $\mathrm{T}$ cell development and that their epigenetic regulation contributes to the transgenerational phenomenon observed here.

\section{Discussion}

The present study documents a rare example of transgenerational inheritance of epimutations in vertebrates, and, to the best of our knowledge, the first case of such a phenomenon that is linked with a specific immunological phenotype. When mutant sperm fertilize an egg produced by wild-type or $d n m t 1^{+/ \mathrm{m}}$ heterozygous females, developmentally programmed changes of DNA methylation patterns during germ cell development occur in the presence of maternally provided dnmt1 protein. Paternal methylomes are known to be stably propagated throughout embryogenesis, and the heavily methylated sperm DNA determines the DNA methylome of the early embryo ${ }^{13-15}$. However, remodeling of an aberrant paternal methylation pattern can still occur, otherwise the low levels of methylation observed in mutant sperm would have been preserved over the course of several generations. As a result of the combined activities of dnmt 1 and other dnmts, such as those with de novo activities, as well as other pathways modulating the DNA methylation pattern in direct or indirect fashion, nearly complete restoration of the original wildtype methylation pattern is achieved in G4 sperm. Hence, it appears that the developmentally programmed changes in DNA methylation patterns are controlled by a rheostat that is blind to the origin of the parental genomes, supporting the notion that the paternal methylation pattern does not serve as a template for the remodeling of the maternal methylome ${ }^{13}$. Our results also indicate that the partially restored methylation pattern appears to exist in two subtly different forms; one of these segregating epialleles fails to support larval $\mathrm{T}$ cell development, the other is seemingly compatible with normal lymphopoiesis. Overall, the differences in DNA methylation between $\mathrm{G}^{+}$and $\mathrm{G} 4 *$ sperm appear limited; in analogy to the situation in plants ${ }^{35,36}$, it may be possible to use epigenetic inbred lines to precisely identify the crucial epigenetic signature that supports larval $\mathrm{T}$ cell development. Unfortunately, at present, methods for the purification of zebrafish lymphoid precursors are not available. Once this becomes possible, it will be interesting to compare the methylation patterns of gametes with those of somatic cells giving rise to the specific phenotype observed here.

Our results support the notion that lymphoid development is particularly sensitive to perturbation of DNA methylation; this phenomenon is not restricted to fish, since the phenotype of impaired lymphopoiesis is reminiscent of that of mice with low expression levels of wild-type protein ${ }^{37,38}$. Collectively, these findings suggest that DNA methylation serves as a mechanism to protect the development of lymphoid cells ${ }^{39}$. Since lymphocytes represent an evolutionarily recent innovation in the haematopoietic system ${ }^{40}$, it attests to the flexibility of epigenetic regulation to integrate evolutionary novelties into pre-existing physiology. Certain immunodeficiency syndromes in animals and humans are caused by genetic alterations of DNMTs ${ }^{41-43}$ or are linked to sporadic epigenetic variations ${ }^{44}$. However, given the differences in the processes of parental reprogramming between fish and mammals, we consider it improbable that the mechanism underlying the particular immunodeficiency disorder described here also applies to so far unexplained immunodeficiency disorders in human patients.

\section{Methods}

Animals. The zebrafish (D. rerio) wild-type strain TLEK (Tüpfel long fin/Ekkwill) is maintained in the animal facility of the Max Planck Institute of Immunobiology and Epigenetics, Freiburg, Germany and was used for crosses with the dnmt1 mutants. The IY071 mutant line has been described ${ }^{23}$, as has been the ikaros:eGFP transgenic reporter line ${ }^{27,28}$. All animal experiments were performed in accordance with relevant guidelines and regulations, approved by the review committee of the Max Planck Institute of Immunobiology and Epigenetics and the Regierungspräsidium Freiburg, Germany (license Az 35-9185.81/G-14/106).

Morphants. Morphants were generated by injection of anti-sense morpholino oligonucleotides (Gene Tools, Philomath, OR) to block translation of both maternal and zygotic mRNAs ("ATG morpholinos"), or to block splicing of zygotic pre-mRNAs ("splice morpholinos"). To block zygotic rptor activity, a morpholino targeting the splice donor site of exon 3 of the rptor gene was used (5'

TGGATGGATGGATGCTCACCTATC; final concentration in injection buffer $0.067 \mathrm{mM}$ ); to inhibit translation of runx3 mRNA, a morpholino overlapping the initiation site was used ( $5^{\prime}$ - ACGGGAATATGCATCACAACAGATT; final concentration in injection buffer $0.133 \mathrm{mM}$ ). Stock solutions of morpholinos were diluted as required in injection buffer $(0.05 \%$ (v/v) phenol red; 1x Danieau Buffer (http://cshprotocols.cshlp.org/content/2011/7/pdb.rec12467.full). Approximately $1-2 \mathrm{~nL}$ of solution were injected into fertilized eggs ${ }^{23}$. The morphants were analyzed at $5 \mathrm{dpf}$ by RNA in situ hybridization using a combination of ragl-and $g h$ specific probes, and the results expressed as a thymopoietic index, a dimensionless number (see below).

Thymopoietic index. Thymic ragl gene expression is a marker of ongoing assembly of $\mathrm{T}$ cell receptor genes. Hence, the intensity of the RNA in situ signal correlates with the number of differentiating $\mathrm{T}$ cells, which we consider to be a measure of $\mathrm{T}$ cell development. In order to provide an internal control (technical, with respect to the hybridization process as such; and, biological, with respect to the tissue specificity of the observed genetic effects), we employed a probe specific for the growth hormone $(g h)$ gene, which marks a subset of cells in the hypophysis. Determination of $\mathrm{ragl} / \mathrm{gh}$ ratios was carried out as follows: after RNA in situ hybridization with ragl and $g h$ probes, ventral images of 4-5 dpf zebrafish larvae were taken on an MZFLIII (Leica) microscope using a digital camera DFC300FX (Leica), essentially generating a two-dimensional projection of the threedimensional structure. The areas of ragl and $g h$ signals were measured using ImageJ (ImageJ 1.52a; available at http://imagej.nih.gov/ij), and the ratio of average of the rag1-positive area vs. $g h$-positive area was calculated as a measure of thymopoietic activity. After photographic documentation of the RNA in situ hybridization signal, larvae were processed for genomic DNA extraction for subsequent genotyping.

RNA extraction and cDNA synthesis. Total RNA was extracted using TRI Reagent (Sigma) following the manufacturer's instructions. After treatment with DNaseI (Promega), RNA extraction using TRI Reagent was repeated. Superscript II Reverse Transcriptase (Invitrogen) and oligo(dT) were used for cDNA synthesis from total RNA. 


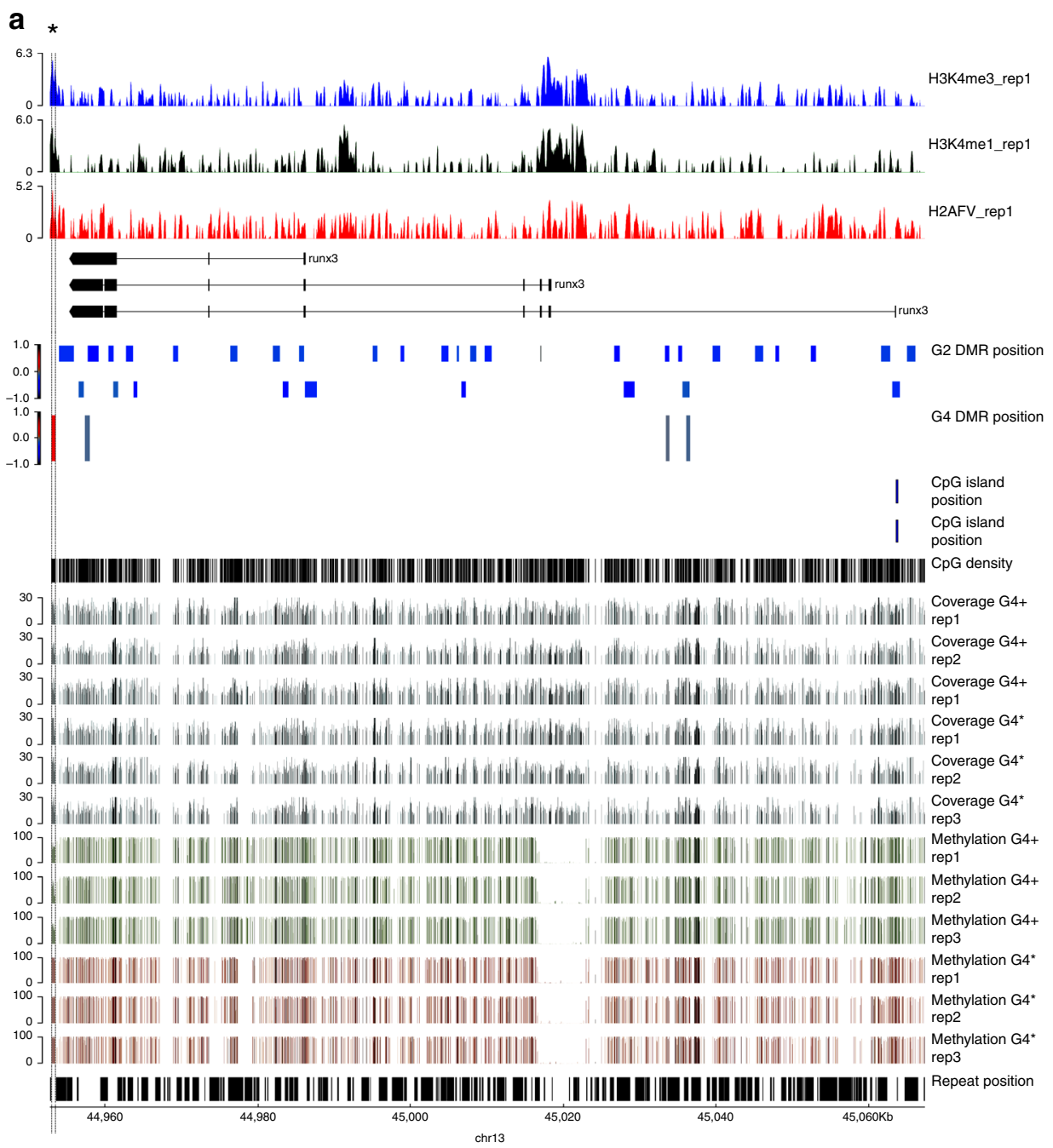

b

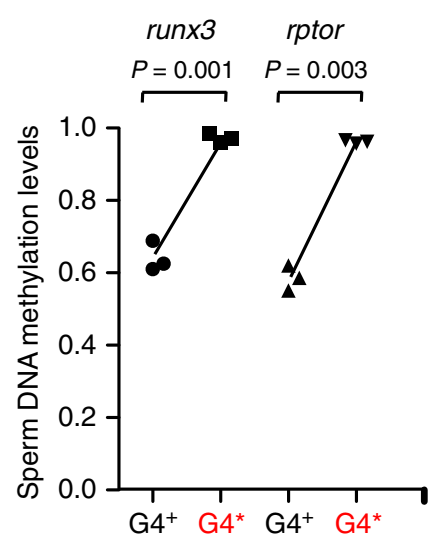

C

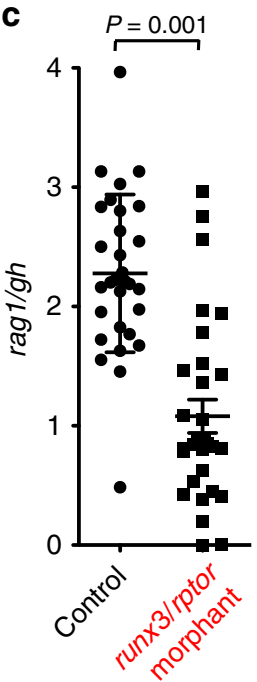

Quantitative PCR. qPCR was carried out using SYBR Premix Ex Taq (Takara) and 7500 fast real-time PCR system (Applied Biosystems) ${ }^{23,45}$. actb1 was used as a reference gene. The primer sets for zebrafish genes were purchased from BioRad (https://www.bio-rad.com/de-de/product/primepcr-pcr-primers-assays-arrays? $\mathrm{ID}=\mathrm{M} 0 \mathrm{HROA15})$. gata2b, qDreCID0018645; $c m y b$, qDreCID0021456; $m p x$, qDreCID0017849; gata1a, qDreCID0013676; cebpa, qDreCED0006492; ikzf1, qDreCID0018943; gata3, qDreCED0006945; rag1, qDreCED0021315; lck, qDreCID0022016; zap70, qDreCID0014002; dnmt1, qDreCED0019976; dnmt3aa, qDreCID0018392; dnmt3ab, qDreCID0019082; dnmt3ba (aka dnmt3b),
qDreCED0021338; dnmt3bb.1 (aka dnmt4), qDreCID0005035; dnmt3bb.2 (aka $d n m t 3$ ), qDreCID0016654; dnmt3bb.3 (aka dnmt5), qDreCED0021863; gadd45aa, qDreCED0006281; gadd45ab, qDreCED0015505; tet1, qDreCED0015074; tet2, qDreCED0010969; tet3, qDreCID0016164; actb1, qDreCED0020462.

Histological analysis. Histological analysis was carried out after formalin fixation, paraffin embedding and hematoxylin/eosin staining of sections ${ }^{46}$. For image 
Fig. 7 Molecular basis of transgenerational inheritance of impaired larval $\mathbf{T}$ cell development. a Characterization of the runx 3 locus. In the first three rows, various chromatin marks are indicated ${ }^{15}$. The structures of known transcripts across the runx 3 locus are shown below. The positions of candidate DMRs called in G2 sperm DNAs are shown underneath the transcript structures, as are the three candidate DMRs discovered in G4+ and G4* sperm DNA. The position of the only annotated runx3 CpG island is indicated above the CpG density track. The filtered coverage across the locus in the three WGBS replicates is indicated as well as the extent of methylation in the $\mathrm{CpGs}$ that were evaluated in the comparison of $\mathrm{G}^{+}$and $\mathrm{G}^{\star}{ }^{\star}$ sperm DNAs. The bottom row indicates the positions of repeat across the locus. Note that the hypermethylated DMR in G4* sperm DNA coincides with a peak in H3K4 methylation. b DNA methylation levels in DMRs associated with runx3 and rptor genes in sperm of G4 males. For runx3, the data pertain to the three replicates of the DMR marked with an asterisk in a; for rptor, the relevant DMR is indicated by an asterisk in Supplementary Fig. 5b. c Combined knock-down of runx3 and rptor by anti-sense oligonucleotides impairs larval T cell development. Each data point represents one animal analyzed at 5 dpf by RNA in situ hybridization. In (b, c), each data point represents one animal; t-test, two-tailed; mean \pm s.e.m. Source data are provided as Source Data file.

analysis of histological sections, JmageJ software was used (ImageJ 1.52a; available at http://imagej.nih.gov/ij).

Flow cytometry. Flow cytometric analysis of light-scatter characteristics of WKM cells was carried out as described by Traver et al. ${ }^{47}$; dead cells were excluded by staining with FluoroGold (Santa Cruz).

Treatment of embryos with Dnmt inhibitors. A stock solution of 5-Aza-2'deoxycytidine (Sigma) was prepared in E3 medium, and diluted to the desired final concentration. Wild-type embryos were exposed to the inhibitor ${ }^{48}$ beginning at 24 hpf for a total of $48 \mathrm{~h}$; at $72 \mathrm{hpf}$, embryos were washed and continuously cultured in $\mathrm{E} 3$ medium.

Whole genome bisulfite sequencing. Genomic DNA was extracted from three $d n m t 1^{+l+}$ and three $d n m t 1^{-1-}$ zebrafish of G2 generation at $18 \mathrm{dpf}$, sperm of three $d n m t 1^{+/+}$and three $d n m t 1^{-/-}$zebrafish of G2 generation, and sperm of three G4* and three G4+ fish of G4 generation using the DNeasy blood and tissue kit (Qiagen). $1 \mu \mathrm{g}$ and $0.5 \mu \mathrm{g}$ of DNA was used for bisulfite reactions and library construction using the TruSeq DNA PCR-free library preparation kit (Illumina) and the EpiGnome Methyl-Seq kit (Epicentre), respectively. The fragments were sequenced in paired-end $100 \mathrm{bp}$ mode on 1 lane of Illumina HiSeq 2500 instrument.

Whole genome bisulfite read alignment. Raw sequencing reads were trimmed with cutadapt version 1.9.1 ref. ${ }^{49}$ as follows: the first two (TruSeq) or six (Epignome) 5'-most nucleotides were hard-trimmed and Illumina adapter sequences removed. Bisulfite-specific operations on reads and reference genome were performed with methylCtools version 0.9 .4 ref. ${ }^{50}$. Bisulfite-converted reads were mapped to bisulfite-converted danRer10 zebrafish genome with bwa-mem version 0.7.12 separately for the two library types. Back-converted bam files were sorted with samtools version 1.3.1, PCR duplicates removed and read group information added with Picard tools v1.136. The two resulting bam files per sample were merged with samtools and methylation bias profiled with MethylDackel v0.1.7 [https://github.com/dpryan79/MethylDackel]. Bam files from this step were further used as inputs for de novo DMR discovery in each dataset, as well as for evaluation of methylation values of target genomic regions provided as bed files (for details, see below).

Extraction of methylation values per CpG. Methylated and unmethylated read counts per $\mathrm{CpG}$ position were extracted with methylCtools v0.9.4 with mapping quality threshold of 10, SNP detection, counting only one of two overlapping paired end reads, skipping 5 nucleotides from each read length and zero-padding of uncovered positions.

Quality filtering of CpGs and data plotting. Data postprocessing was performed in $\mathrm{R}$ version 3.2.3. Raw methylation values were set to $\mathrm{NA}$ for $\mathrm{CpG}$ positions with at least $0.25 \mathrm{SNP}$ allelic frequency as well as for positions with aggregate coverage of less than 10 reads. Mean methylation ratios per $\mathrm{CpG}$ position were calculated as mean over all the replicates per group. Only complete observations were used (positions with any NA values were removed). These group-mean CpG methylation ratios were used to produce density plots in Fig. 1.

Detection of de novo methylated regions (DMRs). Methylation values for single CpG positions (complete cases) were used as input to metilene v 0.2-6 ref. ${ }^{51}$. Wildtype samples were passed in as group A, and mutant as group B. Candidate DMRs detected by metilene were re-evaluated for differential methylation in R. At least $20 \%$ of detected CpGs per DMR were required and at most 1 sample with an NA value was allowed. Methylation values of detected $\mathrm{CpGs}$ were aggregated as mean per interval per replicate sample. Differential methylation was re-evaluated using limma on logit-transformed interval means. DMRs were filtered to retain those with FDR $<5 \%$. Filtered DMRs were further annotated with the distance to the nearest gene using bedtools version 2-2.19.0 and Ensembl release 83 gene models for GRCz10.

Generation of coverage and methylation bigwig files. Bigwig files with coverage and methylation for G2 and G4 datasets were generated by running snakePipes ${ }^{52}$ v1.2.1 with G2 and G4 bam files as input.

Evaluation of G4 sperm DMRs in G2 sperm WGBS data. Bam files obtained through read alignment were re-analyzed with the WGBS workflow in the snakePipes ${ }^{52}$ v1.2.1 modified for the purpose of this manuscript (see, https://github. com/katsikora/snakepipes_fork). Methylation values were extracted from G2 bam files for genomic intervals identifed as hypo- and hypermethylated DMRs in the comparison of the G4* vs G4 + fish sperm, separately. Aggregate methylation values per interval were obtained as above for the DMRs. The matrix of logittransformed methylation values per interval was input to differential analysis with limma version 3.26.9 ref. ${ }^{53}$. Intervals were filtered for absolute difference of at least $20 \%$ between MT and WT fish and FDR $<2 \%$.

Reanalysis of publically available zebrafish ChIP-seq data. Input and ChIP reads for zebrafish sperm H3K4me3, H3K4me1, H2AFV, H3K27ac, H3K27me3, $\mathrm{H} 3 \mathrm{~K} 14 \mathrm{ac}^{15}$ were downloaded from GEO (GSE95033), as were input and ChIP reads for 6.0hpf zebrafish embryo H3K9me3 $3^{54}$, GSE113086). This embryo stage was chosen as there was no sufficient $\mathrm{H} 3 \mathrm{~K} 9 \mathrm{me} 3$ signal in the earlier embryo stages $^{54}$. Reads were mapped to zebrafish genome danRer10 and processed with snakePipes ${ }^{52}$ version 1.3.0 DNA-mapping and ChIP-seq workflows to produce bigwig files with $\log 2$ ratio ChIP over input.

Reanalysis of publically available zebrafish embryo HiC data. $\mathrm{HiC}$ reads for $24 \mathrm{hpf}$ embryos ${ }^{55}$ were downloaded from GEO (GSE105013) and processed with snakePipes 1.3.0 HiC workflow to produce $\mathrm{HiC}$ matrices at $20 \mathrm{~kb}$ resolution and to call TAD positions.

runx3 and raptor genomic tracks plots. Genomic tracks plot was obtained with pyGenomeTracks ${ }^{56}$ version 3.2. Used were $\log 2$ ratio ChiP over input bigwig files obtained for H3K4me3, H3K4me1 and H2AFV marks as described above, Ensembl version 88 gene model gtf for GRCz10, bed files with G2 and G4 DMR positions, bed file with CpG island position for danRer10 (UCSC), bed file with positions of all $\mathrm{CpGs}$ in the reference genome ("CpG density"), bigwig files with filtered $\mathrm{CpG}$ coverage for G4+ and G4* replicates, bigwig files with methylation value $(0-100 \%$ ) for G4+ and G4* replicates, and a repeat masker bed file for danRer10 (UCSC). Dashed vertical lines highlight the position of the single DMR differentially methylated (hypermethylated) in G4* vs G4+ fish. The runx3 plot was generated for genomic interval chr13:44952862-45067319 and the rptor plot for the genomic interval chr6:17422590-17702769, covering the gene locus and adding $3 \mathrm{~kb}$ flanking regions on each side of it.

Methylation values for gene regulatory elements. Genomic intervals for gene regulatory features were obtained with the genFeatures function of the BioConductor R package systemPipeR ${ }^{57}$ v1.6.2. Input was Ensembl release $83 \mathrm{gtf}$ file and GRCz10 chromosome sizes. The genome was segmented into promoters, introns, exons, and intergenic regions. Promoters were defined as 500nt upstream to transcriptional start site, irrespectively of any positionwise overlap with another feature. The other three feature types were further reduced to disjoint ranges i.e. nonoverlapping any other feature, and of length at least $5 \mathrm{nt}$. $\mathrm{CpG}$ island positions for danRer10 from UCSC were used as is. To obtain genomic intervals for placeholder nucleosomal regions, H2AZ and H3K4me1 ChIP-seq peaks ${ }^{15}$ downloaded as bed files from GEO (GSE95030) were intersected. Intersecting regions of at least 100 nt length were used in further analysis. Bed files with genomic intervals specified above were input to modified snakePipes WGBS v.1.2.1 workflow, and evaluated in both G2 and G4 datasets to obtain mean methylation ratios per interval and violin plots. 
Methylation values for developmental DMRs. Genomic positions for zebrafish developmental DMRs (24hpf vs epiboly, $48 \mathrm{hpf}$ vs $24 \mathrm{hpf}$, hypo- and hypermethylated) were downloaded from Supplementary materials of the original publication ${ }^{32}$ and lifted-over to danRer10 genome using the UCSC lift-over web tool. DMR lists were input to snakePipes v.1.2.1 and evaluated in both G2 and G4 datasets to obtain mean methylation ratios per interval and violin plots.

Methylation values for zebrafish genomic repeats. Repeat masker file for danRer10 from UCSC was converted to bed file, and used as input to snakePipes v.1.2.1 to evaluate in both G2 and G4 datasets and obtain mean methylation ratios per interval and density plots. Methylation extraction for genomic repeats was done with the same threshold on mapping quality as for the other target intervals (at least 10).

\section{Coincidence of DMRs with chromosome-level genomic features. Genomic} tracks for chromosomes 6 and 7 were produced with pyGenomeTracks ${ }^{56}$ version 3.2. Used were $\mathrm{HiC}$ matrices for $24 \mathrm{hpf}$ embryo and bed file with TAD positions for 24hpf embryo obtained as described in sections above, $\log 2$ ratio ChiP over input bigwig files obtained for H3K4me3, H3K27me3 and H3K9me3 marks as described in sections above, bigwig file with filtered CpG coverage for one G4 replicate, bed files with hypo- and hypermethylated G4 DMR positions, bed file with CpG positions (UCSC) and bed file with repeat positions (UCSC). Dashed vertical lines show TAD boundaries obtained as described in sections above.

Coincidence of histone modification with G4 DMRs. Bigwig files of $\log 2$ ratio ChiP over input obtained for H3K3me1, H3K4me3, H3K27me3, H2AFV, and $\mathrm{H} 3 \mathrm{k} 14 \mathrm{ac}$ for merged replicates were analyzed with deepTools v3.3.1. ChIP signal aggregated over $10 \mathrm{nt}$ bins was extracted for unchanged, hypo- and hypermethylated G4 DMRs, rescaled to $500 \mathrm{nt}$ with $100 \mathrm{nt}$ added on each flank, to produce a matrix with DMRs in rows and bins in columns, grouped by chromatin mark and DMR source. Profile plots of $\log 2$ ratio ChIP signal over input with 0.95 confidence intervals were obtained for the DMR groups using deepStats v0.4 ref. ${ }^{58}$ with 1000 bootstraps, as were binwise Wilcoxon-test $\mathrm{P}$ value plots. A cutoff adjusted $P$ value of 0.05 was used.

Statistical methods. No randomization of animals was done in the present studies; phenotypes were recorded by a blinded observer before genotyping. No animals were excluded from analyses. Samples size was estimated from the degrees of variability in previous analyses ${ }^{23,46}$ in order to be able to detect biologically meaningful differences in examined parameters, usually $20 \%$ difference from control values. t-tests were performed for samples with equal variance; otherwise, F-tests were used. Other statistical procedures are detailed in the respective sections above.

Reporting summary. Further information on research design is available in the Nature Research Reporting Summary linked to this article.

\section{Data availability}

The original sequencing data have been deposited in the GEO database and are available under accession number "GSE98647". All other relevant data supporting the key findings of this study are available within the article and its Supplementary Information files or from the corresponding author upon reasonable request. Source data are provided with this paper. A reporting summary for this Article is available as a Supplementary Information file. Source data are provided with this paper

\section{Code availability}

The R code necessary to reproduce the statistical analyses and results is reported in Supplemental Code and is available at https://github.com/katsikora/ Iwanami2019_SupplementaryCodeAndData_A.

Received: 28 October 2019; Accepted: 11 August 2020;

Published online: 09 September 2020

\section{References}

1. Skvortsova, K., Iovino, N. \& Bogdanovic, O. Functions and mechanisms of epigenetic inheritance in animals. Nat. Rev. Mol. Cell. Biol. 19, 774-790 (2018).

2. Perez, M. F. \& Lehner, B. Intergenerational and transgenerational epigenetic inheritance in animals. Nat. Cell. Biol. 21, 143-151 (2019).

3. Heard, E. \& Martienssen, R. A. Transgenerational epigenetic inheritance: myths and mechanisms. Cell 157, 95-109 (2014).

4. Jones, P. A. Functions of DNA methylation: islands, start sites, gene bodies and beyond. Nat. Rev. Genet. 13, 484-492 (2012).

5. Schubeler, D. Function and information content of DNA methylation. Nature 517, 321-326 (2015)
6. Luo, C., Hajkova, P. \& Ecker, J. R. Dynamic DNA methylation: In the right place at the right time. Science 361, 1336-1340 (2018).

7. Hackett, J. A. \& Surani, M. A. Beyond DNA: programming and inheritance of parental methylomes. Cell 153, 737-739 (2013).

8. Seisenberger, S. et al. The dynamics of genome-wide DNA methylation reprogramming in mouse primordial germ cells. Mol. Cell 48, 849-862 (2012).

9. Guibert, S., Forne, T. \& Weber, M. Global profiling of DNA methylation erasure in mouse primordial germ cells. Genome Res. 22, 633-641 (2012).

10. Smith, Z. D. et al. DNA methylation dynamics of the human preimplantation embryo. Nature 511, 611-615 (2014).

11. Gkountela, S. et al. DNA demethylation dynamics in the human prenatal germline. Cell 161, 1425-1436 (2015).

12. Radford, E. J. et al. In utero effects. In utero undernourishment perturbs the adult sperm methylome and intergenerational metabolism. Science 345, 1255903 (2014).

13. Potok, M. E., Nix, D. A., Parnell, T. J. \& Cairns, B. R. Reprogramming the maternal zebrafish genome after fertilization to match the paternal methylation pattern. Cell 153, 759-772 (2013).

14. Jiang, L. et al. Sperm, but not oocyte, DNA methylome is inherited by zebrafish early embryos. Cell 153, 773-784 (2013).

15. Murphy, P. J., Wu, S. F., James, C. R., Wike, C. L. \& Cairns, B. R. Placeholder nucleosomes underlie germline-to-embryo DNA methylation reprogramming. Cell 172, 993-1006.e1013 (2018).

16. Ortega-Recalde, O., Day, R. C., Gemmell, N. J. \& Hore, T. A. Zebrafish preserve global germline DNA methylation while sex-linked rDNA is amplified and demethylated during feminisation. Nat. Commun. 10, 3053 (2019).

17. Skvortsova, K. et al. Retention of paternal DNA methylome in the developing zebrafish germline. Nat. Commun. 10, 3054 (2019).

18. Smith, Z. D. \& Meissner, A. DNA methylation: roles in mammalian development. Nat. Rev. Genet. 14, 204-220 (2013).

19. Li, E., Bestor, T. H. \& Jaenisch, R. Targeted mutation of the DNA methyltransferase gene results in embryonic lethality. Cell 69, 915-926 (1992).

20. Anderson, R. M. et al. Loss of Dnmtl catalytic activity reveals multiple roles for DNA methylation during pancreas development and regeneration. Dev. Biol. 334, 213-223 (2009).

21. Lee, P. P. et al. A critical role for Dnmt1 and DNA methylation in T cell development, function, and survival. Immunity 15, 763-774 (2001).

22. Rai, K. et al. Zebra fish Dnmt1 and Suv39h1 regulate organ-specific terminal differentiation during development. Mol. Cell. Biol. 26, 7077-7085 (2006).

23. Iwanami, N. et al. Forward genetic screens in zebrafish identify pre-mRNAprocessing pathways regulating early T cell development. Cell Rep. 17, 2259-2270 (2016).

24. Boehm, T., Bleul, C. C. \& Schorpp, M. Genetic dissection of thymus development in mouse and zebrafish. Immunol. Rev. 195, 15-27 (2003).

25. Takeshita, K. et al. Structural insight into maintenance methylation by mouse DNA methyltransferase 1 (Dnmt1). Proc. Natl Acad. Sci. USA 108, 9055-9059 (2011).

26. Song, J., Teplova, M., Ishibe-Murakami, S. \& Patel, D. J. Structure-based mechanistic insights into DNMT1-mediated maintenance DNA methylation. Science 335, 709-712 (2012)

27. Bajoghli, B. et al. Evolution of genetic networks underlying the emergence of thymopoiesis in vertebrates. Cell 138, 186-197 (2009).

28. Hess, I. \& Boehm, T. Intravital imaging of thymopoiesis reveals dynamic lympho-epithelial interactions. Immunity 36, 298-309 (2012).

29. Tian, Y. et al. The first wave of T lymphopoiesis in zebrafish arises from aorta endothelium independent of hematopoietic stem cells. J. Exp. Med. 214, 3347-3360 (2017).

30. Rai, K. et al. DNA demethylation in zebrafish involves the coupling of a deaminase, a glycosylase, and gadd45. Cell 135, 1201-1212 (2008).

31. Santi, D. V., Norment, A. \& Garrett, C. E. Covalent bond formation between a DNA-cytosine methyltransferase and DNA containing 5-azacytosine. Proc. Natl Acad. Sci. USA 81, 6993-6997 (1984).

32. Bogdanovic, O. et al. Active DNA demethylation at enhancers during the vertebrate phylotypic period. Nat. Genet. 48, 417-426 (2016).

33. Taniuchi, I. et al. Differential requirements for Runx proteins in CD4 repression and epigenetic silencing during $\mathrm{T}$ lymphocyte development. Cell 111, 621-633 (2002).

34. Hoshii, T. et al. Loss of mTOR complex 1 induces developmental blockage in early T-lymphopoiesis and eradicates T-cell acute lymphoblastic leukemia cells. Proc. Natl Acad. Sci. USA 111, 3805-3810 (2014).

35. Johannes, F. et al. Assessing the impact of transgenerational epigenetic variation on complex traits. PLoS Genet. 5, e1000530 (2009).

36. Cortijo, S. et al. Mapping the epigenetic basis of complex traits. Science $\mathbf{3 4 3}$, 1145-1148 (2014)

37. Gaudet, F. et al. Induction of tumors in mice by genomic hypomethylation. Science 300, 489-492 (2003). 
38. Broske, A. M. et al. DNA methylation protects hematopoietic stem cell multipotency from myeloerythroid restriction. Nat. Genet. 41, 1207-1215 (2009).

39. Greenberg, M. V. C. \& Bourc'his, D. The diverse roles of DNA methylation in mammalian development and disease. Nat. Rev. Mol. Cell Biol. 20, 590-607 (2019).

40. Boehm, T. Design principles of adaptive immune systems. Nat. Rev. Immunol. 11, 307-317 (2011)

41. Hansen, R. S. et al. The DNMT3B DNA methyltransferase gene is mutated in the ICF immunodeficiency syndrome. Proc. Natl Acad. Sci. USA 96, 14412-14417 (1999).

42. Xu, G. L. et al. Chromosome instability and immunodeficiency syndrome caused by mutations in a DNA methyltransferase gene. Nature 402, 187-191 (1999).

43. Campos-Sanchez, E., Martinez-Cano, J., Del Pino Molina, L., Lopez-Granados, E. \& Cobaleda, C. Epigenetic deregulation in human primary immunodeficiencies. Trends Immunol. 40, 49-65 (2019).

44. Rodriguez-Cortez, V. C. et al. Monozygotic twins discordant for common variable immunodeficiency reveal impaired DNA demethylation during naiveto-memory B-cell transition. Nat. Commun. 6, 7335 (2015).

45. Iwanami, N. et al. Genetic evidence for an evolutionarily conserved role of IL-7 signaling in T cell development of zebrafish. J. Immunol. 186, 7060-7066 (2011).

46. Schorpp, M. et al. Conserved functions of Ikaros in vertebrate lymphocyte development: genetic evidence for distinct larval and adult phases of $\mathrm{T}$ cell development and two lineages of B cells in zebrafish. J. Immunol. 177, 2463-2476 (2006).

47. Traver, D. et al. Transplantation and in vivo imaging of multilineage engraftment in zebrafish bloodless mutants. Nat. Immunol. 4, 1238-1246 (2003).

48. Martin, C. C., Laforest, L., Akimenko, M. A. \& Ekker, M. A role for DNA methylation in gastrulation and somite patterning. Dev. Biol. 206, 189-205 (1999).

49. Martin, M. Cutadapt removes adapter sequences from high-throughput sequencing reads. EMBnet. J. 17, 10-12 (2011).

50. Hovestadt, V. et al. Decoding the regulatory landscape of medulloblastoma using DNA methylation sequencing. Nature 510, 537-541 (2014).

51. Juhling, F. et al. metilene: fast and sensitive calling of differentially methylated regions from bisulfite sequencing data. Genome Res. 26, 256-262 (2016).

52. Bhardwaj, V. et al. snakePipes: facilitating flexible, scalable and integrative epigenomic analysis. Bioinformatics 35, 4757-4759 (2019).

53. Ritchie, M. E. et al. limma powers differential expression analyses for RNAsequencing and microarray studies. Nucleic Acids Res 43, e47 (2015).

54. Laue, K., Rajshekar, S., Courtney, A. J., Lewis, Z. A. \& Goll, M. G. The maternal to zygotic transition regulates genome-wide heterochromatin establishment in the zebrafish embryo. Nat. Commun. 10, 1551 (2019).

55. Kaaij, L. J. T., van der Weide, R. H., Ketting, R. F. \& de Wit, E. Systemic loss and gain of chromatin architecture throughout Zebrafish development. Cell Rep. 24, 1-10.e14 (2018).

56. Ramirez, F. et al. High-resolution TADs reveal DNA sequences underlying genome organization in flies. Nat. Commun. 9, 189 (2018).

57. TW, H. B. \& Girke, T. systemPipeR: NGS workflow and report generation environment. BMC Bioinforma. 17, 388 (2016).

58. Gautier, R. gtrichard/deepStats: deepStats 0.3.1 (Version 0.3.1), https://doi. org/10.5281/zenodo.3336593 (2019).

59. Schorpp, M. et al. A zebrafish orthologue (whnb) of the mouse nude gene is expressed in the epithelial compartment of the embryonic thymic rudiment. Mech. Dev. 118, 179-185 (2002).
60. Nehls, M., Pfeifer, D., Schorpp, M., Hedrich, H. \& Boehm, T. New member of the winged-helix protein family disrupted in mouse and rat nude mutations. Nature 372, 103-107 (1994).

\section{Acknowledgements}

These studies received funding from the Max Planck Society, and the European Research Council (ERC) under the European Union's Seventh Framework Programme (FP7/ 2007-2013), ERC Grant agreement 323126 (all to T.B.), and were partially supported by Platform Project for Supporting Drug Discovery and Life Science Research (Basis for Supporting Innovative Drug Discovery and Life Science Research [BINDS]) from AMED under Grant Number JP19am0101070 (support number 1563) (to K.T.)

\section{Author contributions}

N.I., D.-F.L., C.O'M. and M.S., C.O'M. designed and performed experiments. K.T. provided structural data. K.S. conducted bioinformatic analyses. All authors analyzed data, discussed results and reviewed the manuscript. N.I. and T.B. wrote the manuscript.

\section{Funding}

Open Access funding provided by Projekt DEAL.

\section{Competing interests}

The authors declare no competing interests.

\section{Additional information}

Supplementary information is available for this paper at https://doi.org/10.1038/s41467020-18289-9.

Correspondence and requests for materials should be addressed to T.B

Peer review information Nature Communications thanks the anonymous reviewers for their contribution to the peer review of this work.

Reprints and permission information is available at http://www.nature.com/reprints

Publisher's note Springer Nature remains neutral with regard to jurisdictional claims in published maps and institutional affiliations.

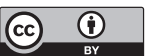

Open Access This article is licensed under a Creative Commons Attribution 4.0 International License, which permits use, sharing, adaptation, distribution and reproduction in any medium or format, as long as you give appropriate credit to the original author(s) and the source, provide a link to the Creative Commons license, and indicate if changes were made. The images or other third party material in this article are included in the article's Creative Commons license, unless indicated otherwise in a credit line to the material. If material is not included in the article's Creative Commons license and your intended use is not permitted by statutory regulation or exceeds the permitted use, you will need to obtain permission directly from the copyright holder. To view a copy of this license, visit http://creativecommons.org/ licenses/by/4.0/.

(c) The Author(s) 2020 